\title{
PSYCHO-HYGIENIC COMPETENCE OF SPECIALISTS IN INTERNET- ENVIRONMENT USE
}

\author{
Sergiy Boltivets \\ Grigory Kostyuk Psychological \\ Institute of the Ukrainian National Academy of Pedagogical Sciences, Ukraine \\ E-mail: boltivetssergij@i.ua
}

The awareness by the "man-man" professions specialists of their own psycho-hygienic skills and competence content, which are formed in the process of professionalization, is determined by conceptual ideas. They are developed within a certain people-study profession and cover use, interaction and reflection of a personality in the Internet environment.

We have introduced into scientific use the category of mental health quality which represents the individual property of personality to ensure his/her own procedural integrity, that is adequate to internal nature, in cooperation with own selfness and environment. This category certainly includes the continuingly improving means of both learning the world and self-implementation in it including the Internet-environment.

The principal place among the psychological researches of Internet use effects in human life was taken by the searches of harms in the form of addictions: dependence on the Internet, Internetaddiction, pathological, patho-characterological or problem Internet use, etc.

At the same time, in our opinion, the personality formation, human personal development in ontogeny (that is his/her lifelong development) is a process of forming a certain type of mental health quality, since a product and a result of this process are the creation of a certain system of psychic self-organization and the individual self-realization. We cannot imagine how the human civilization development would be if this system of the personal psychic self-organization and self-realization means did not include the total learning of writing, reading and other ways of culture reception which is the world experience exchange between different generations and between people within one generation. Therefore, alienation, fragmentation or non-development - let's call it as a vacuum of Internet competence - in the educational growth of personality change the structure of the personal mental self-organization in a certain social formation. The mentioned discrepancy creates preconditions for the mental trauma to a person for the following age periods of life. Immaturity of the mentioned properties of the mental self-organization determines the structure of the mental health quality that is characterized by lower activity, lowered self-esteem and confidence in abilities and harassment level, and psychological security. Obviously, this is not the equivalent for the symptoms of mental disorder or disease and their components; that allows recommending to compensate the mentioned properties of personal mental self-organization by psychological (developmental, cor- 
rectional, rehabilitation) and pedagogical (teaching and upbringing) methods.

Psycho-hygienic components of the competences of the "man-man" professions specialists in this context should also include:

- Ways to use Internet-competences as means of establishing, expanding or restoring person's social relations in an imaginary (virtual) world that is perceived as a desirable one,

- Ways of formation the ability to self-regulation which is based on the psycho-hygienic principle of balance between imaginary and real in their relationship.

In our view, the problem of Internet-addiction as well as much less perceived problem of man's Internet-competences vacuum equally should have a professional representation of the content of professional competence of the "man-man" professions specialists, and especially - medical and educational psychologists, secondary school teachers and university professors, educators, medical and social workers.

In this professional representation the developing self-control methods as a capacity for the coordinated emotional and volitional function by the main indicators: integrity, rhythm, and plasticity, has a particular importance. At the same time, the specialists of people-study professions should be aware that of the greatest value is those universal forms and patterns of the personal mental selforganization, which are not lost and transformed for the following age periods of human life, and become the tools of learning the changing world. The learning tools belong to a constant process of a person's self-improvement based on the integrity of self-discovery in the world in order to implement their own life meaning - own conscious predestination. Coming from that, the Internetcompetences are means of expanding person's notions which are necessary for individual choice of lifestyle, and therefore have a significant importance for the health and, in particular, mental health. The researchers draw attention to the development of Internet-addiction which, in our opinion, should be considered in procedural terms; as the period needed to compensate the lost or non-developed person's relations with the real world.

Maintenance of balance and coordination of the environmental influences, which include the man-made Internet-environment, is an important task for the psycho-hygienic mission of the peopleoriented occupations specialists. These tasks provide integrity of personality sense-creation in the world, an idea of which is becoming more and more significant as a part of our civilization.

Thus, the development of psycho-hygienic competences of the "man-man" type occupations specialists creates the conditions for realization of the important principles of balance and coordination of the environmental impacts, ideal-realistic unity, and esteem approach in determination of the Internet-influences on personality. 